\title{
Punitions corporelles et actes de brutalité dans les écoles primaires publiques du département de la Seine (1880-1914)
}

Corporal punishment and acts of brutality in state primary schools in the Département de la Seine (1880-1914)

Körperliche Züchtigungen und sonstige Gewaltanwendungen in den öffentlichen Volksschulen des Departements Seine (1880-1914)

Castigos corporales y actos de brutalidad en las escuelas primarias públicas del departamento del Sena (1880-1914)

\section{Jérôme Krop}

\section{(2) OpenEdition}

\section{Journals}

\section{Édition électronique}

URL : https://journals.openedition.org/histoire-education/542

DOI : 10.4000/histoire-education.542

ISSN : 2102-5452

\section{Éditeur}

ENS Éditions

\section{Édition imprimée}

Date de publication : 1 avril 2008

Pagination : 109-132

ISBN : 978-2-7342-1117-4

ISSN : 0221-6280

\section{Référence électronique}

Jérôme Krop, «Punitions corporelles et actes de brutalité dans les écoles primaires publiques du département de la Seine (1880-1914) », Histoire de l'éducation [En ligne], 118 | 2008, mis en ligne le 01 janvier 2013, consulté le 20 mai 2021. URL : http://journals.openedition.org/histoire-education/542 ; DOI : https://doi.org/10.4000/histoire-education.542 


\section{Punitions corporelles et actes de brutalité}

dans les écoles primaires publiques du département

de la Seine (1880-1914)

Jérôme KROP

L'école républicaine est une des institutions les plus emblématiques des débuts de la Troisième République. Elle occupe d'ailleurs une place centrale dans l'imaginaire politique républicain. La figure de l'instituteur de la communale, instructeur et éducateur du peuple, est consacrée, alors que les lois Ferry, puis la loi Goblet, établissent le cadre législatif nécessaire à la constitution de l'école gratuite, laïque et obligatoire. La croyance dans les vertus de l'instruction, dans la capacité de l'école à contribuer à l'instauration d'une République durable, est largement partagée à cette époque. L'école républicaine réussit ainsi à incarner la concrétisation de l'idéal républicain au travers de la figure de l'instituteur. Mais la création de cette école, notamment à travers la progressive laïcisation du personnel scolaire et la constitution d'un corps d'instituteurs et d'institutrices fonctionnaires d'État, est l'aboutissement d'un processus séculaire. Les enseignants s'émancipent de la tutelle de l'Église et, dans une certaine mesure, de celle des autorités locales, alors que, dans chaque département, l'inspecteur d'académie, directeur de l'enseignement primaire, et les inspecteurs primaires sont chargés, sans doute plus qu'ils ne l'ont jamais été, du contrôle de l'application par les enseignants des principes, méthodes et programmes définis par le pouvoir politique. 
L'étude des dossiers des instituteurs et institutrices ayant commencé à enseigner dans la Seine entre 1870 et $1886^{1}$ permet d'appréhender les représentations et les pratiques dans l'enseignement primaire de ce département à cette époque. Entre toutes les informations que renferment ces dossiers (parcours scolaire et professionnel antérieurs, diplômes, mobilité géographique, rémunération), celles qu'apportent les rapports d'enquête des inspecteurs primaires présentent sans doute le plus grand intérêt. Ils informent notamment sur les relations, souvent conflictuelles, entre les directeurs d'école et leurs adjoints et entre les enseignants et les municipalités. Les courriers concernant les situations de conflit entre les acteurs de la vie scolaire donnent lieu à des enquêtes très poussées de la part des inspecteurs. Ceux-ci recueillent les témoignages des autorités locales, des parents, des élèves et, bien sûr, des enseignants. Ces conflits, liés par exemple à la question de la laïcité scolaire, ou les dénonciations dont les enseignants sont l'objet, notamment au sujet de punitions corporelles, peuvent apparaitre anecdotiques. Cependant, leur fréquence atteste de leur importance dans la vie scolaire à la fin du XIX ${ }^{\mathrm{e}}$ ou au début du XX $\mathrm{X}^{\mathrm{e}}$ siècle $^{2}$.

Les plaintes pour recours à des punitions corporelles ou pour faits de brutalité envers les élèves constituent, qu'elles soient fondées ou non, le premier motif des enquêtes des inspecteurs primaires. Soixante-dix plaintes ont été recensées dans les dossiers dépouillés ${ }^{3}$, correspondant à soixante individus (10\% des dossiers étudiés), certains ayant fait l'objet de plusieurs plaintes pendant leur carrière. Dans le cadre de cet article, quarante-quatre plaintes, considérées comme fondées après enquête des inspecteurs, ont été prises en compte. Elles concernent quarante instituteurs et institutrices. Cette petite minorité d'enseignants $(6,7 \%$ des dossiers) doit être évaluée à la lumière des faits incriminés : ce sont souvent des brutalités graves, qui entraîneraient aujourd'hui des poursuites pénales, la

1 Les carrières des instituteurs de notre corpus s'étendent essentiellement jusqu'au premier conflit mondial. Il s'agit donc de la génération des instituteurs qui ont connu la mise en œuvre de la nouvelle législation républicaine. Aussi, les dossiers étudiés concernent l'ensemble de la période qui s'étend de leur entrée dans l'enseignement jusqu'à 1914, même si les événements relatés sont concentrés dans les premières décennies de leurs carrières.

2 Toute étude de l'usage de la violence par les maîtres dans les écoles se situe nécessairement dans le prolongement de l'ouvrage de Jean-Claude Caron, A l'école de la violence, Paris, Aubier, 1999, qui a notamment étudié les archives judiciaire sous le Second Empire.

3 Menée dans le cadre de la préparation de notre thèse sous la direction de Jean-Noël Luc à l'Université de Paris-IV, notre étude repose sur l'exploitation de 660 dossiers d'instituteurs ou institutrices, qui représentent $15 \%$ de la population étudiée (4404 dossiers conservés aux archives de Paris dans la série D1T1). Cet échantillon a été tiré au sort par l'ordinateur en respectant, par rapport à la population totale, la répartition par sexe et la proportion par année de recrutement de 1870 à 1886 . Actuellement, 598 dossiers ont été exploités et constituent la population de référence pour cet article. 
plupart ayant laissé des traces visibles sur le corps de l'élève. En outre, cette violence est fortement sexuée : parmi ces quarante enseignants, trente-deux sont des hommes, soit 11,5\% des dossiers d'instituteurs déjà exploités ; 2,5 \% seulement des institutrices du corpus font l'objet de ce type de plainte ${ }^{4}$.

Ainsi, une minorité non négligeable des instituteurs de la population étudiée a fait l'objet de plaintes, le plus souvent pour de graves faits de violence envers un ou plusieurs élèves, alors même que les règlements scolaires interdisent totalement le recours à des châtiments corporels. Aussi est-il nécessaire d'étudier cette violence, à la lumière des sources, pour en comprendre les ressorts sociaux et la replacer dans ses rapports avec le fonctionnement de l'institution scolaire et dans le contexte global de la société, notamment en étudiant les commentaires des parents et les réactions des autorités scolaires à la demande sociale que constituent les plaintes dénonçant ces pratiques.

\section{I - Des corps marqués par la violence}

Les actes de brutalité peuvent être hiérarchisés en fonction de leur violence et de la gravité des traces qu'ils ont laissées sur le corps des enfants. Certains témoignages présentent l'humiliation de l'élève comme méthode disciplinaire. Par exemple, Berthe Patard laisse une élève, qui aurait fait trop de bruit en entrant dans sa classe, se tenir sur un pied ${ }^{5}$. Un rapport de la délégation cantonale signale en octobre 1891, à propos de Paul Audiard, qu'

en montant en classe avec ses élèves, il n'a pu s'empêcher de donner une taloche au jeune Huret qui n'observait pas la discipline. Je dis " taloche ", mais M. Audiard affirme n'avoir fait que toucher avec la main, sans aucune brutalité, la tête de l'élève Huret ${ }^{6}$.

En décembre de la même année, Alice Dubois fait l'objet d'une plainte alors qu'" elle s'était contentée de frapper un élève avec un cahier composé de quelques feuilles sans couverture ${ }^{7}$.

4 Voir Anne-Marie Sohn, Chrysalides. Femmes dans la vie privée (XIX ${ }^{e}-X X^{e}$ siècle), Paris, Publications de la Sorbonne, 1996, p. 411-421, qui souligne le caractère sexué de l'application des punitions corporelles, majoritairement masculines, mais dans une moindre mesure que dans nos sources. Cela tient peut-être au fait que les coups ne laissant pas de traces sur le corps de l'enfant, provoquent rarement des plaintes officielles, sous-estimant ainsi les punitions corporelles féminines.

5 D1T1 262. Nous indiquons les références de chaque dossier la première fois qu'ils sont cités.

6 D1T1 346.

$7 \quad$ D1T1 157. 
Plusieurs cas relèveraient presque de l'accident si un geste trop brutal de l'instituteur n'en était la cause. Ainsi, en juillet 1886, le directeur d'une école déclare que

les faits relatés dans la lettre de M. Mosnier, père de l'enfant, sont à peu près exacts. Voici ce qui s'est passé : mardi dernier, M. Godard a poussé le jeune Mosnier un peu rudement ; celui-ci s'est heurté la tête contre le tableau noir et s'est fait une bosse au front. J'ai soigné l'enfant et le soir à 4 h., la bosse avait disparu ${ }^{8}$.

Ces situations sont parfois très ambiguës. Ainsi, en juillet 1880, Louis Normand a voulu souffleter un enfant qui lui désobéissait "gravement ", et ce dernier, en essayant d'éviter le coup, est allé se cogner fortement à l'angle d'une table ${ }^{9}$. En mars 1886, le directeur de l'école de la rue de Tolbiac informe ainsi l'inspecteur du $13^{\mathrm{e}}$ arrondissement :

Un de nos élèves, le jeune Valette Louis, âgé de 8 ans, est tombé cette après-midi en classe sur le pied d'une table et s'est fait à la tête une large et profonde blessure.

J'ai fait appeler immédiatement M. le Dr Mangenot, médecin inspecteur de l'école.

Voici, d'après M. Scellos, maître de la $4^{\mathrm{e}}$ classe à laquelle appartient l'enfant blessé, comment l'accident serait arrivé :

"Nous rentrions en classe (après la récréation), le jeune Valette s'étant écarté des rangs, malgré ma défense, je l'ai saisi par le bras pour l'y faire rentrer ; il a perdu l'équilibre en se retirant de mes mains et c'est alors qu'il est tombé contre la table ".

L'enfant prétend, au contraire, avoir été poussé par le maître ${ }^{10}$.

Il arrive que les inspecteurs soient les témoins directs des punitions corporelles. Ainsi, en 1896, l'inspecteur surprend François Cartier pendant la correction d'une dictée :

Au moment où j'entrais, M. Cartier faisait corriger simultanément une dictée et pour marquer la cadence frappait avec sa règle tantôt sur la table, tantôt sur la tête des élèves. [...] Aujourd'hui, à trois heures, il n'avait donné aucune mauvaise note, aucun bon point ; mais il avait mis deux élèves à la retenue, plusieurs au piquet dans la classe et frappé de la main environ 15 enfants $^{11}$.

\footnotetext{
8 D1T1 144.

9 D1T1 59.

10 D1T1 379.

11 D1T1 37.
} 
Cependant, dans la plupart des cas, les plaintes sont motivées par un acte de violence ayant laissé une trace visible sur le corps de l'enfant, trace constituant une preuve susceptible de justifier la plainte. Il arrive qu'à la suite d'une plainte pour une punition un peu plus brutale qu'à l'accoutumée, l'inspecteur découvre une classe dans laquelle les punitions corporelles sont une pratique quotidienne. En janvier 1893, l'inspecteur Drouard note dans son rapport :

J'ai eu l'occasion de relever ce que je savais déjà, hélas, que M. Debroise, à propos de fautes très légères, frappait fréquemment les élèves soit avec la main, soit avec la règle. Dans sa classe même, pendant le mois de décembre, plus de 20 enfants ont été l'objet de punitions corporelles de sa part ${ }^{12}$.

Alors que les plaintes se sont multipliées contre un instituteur de l'école communale de Villemomble en mai-juin 1889, l'inspecteur primaire d'Ollendon signale la généralisation des punitions corporelles dans sa classe :

L'enquête, tout en montrant que, là encore, la passion avait considérablement exagéré les faits, a établi que les châtiments corporels étaient fréquents dans la classe de M. Frégnac. Ce dernier a reconnu immédiatement qu'il lui était arrivé de donner quelques gifles aux enfants, mais malheureusement tout montre, dans l'enquête, que cette mauvaise habitude était de tous les jours, pour ne pas dire de tous les instants. Tous les élèves que j'ai entendus (au nombre de sept : les jeunes Brocard, Lefèvre, Deguin, Roger, Lepère, Jeandot, Loison) ont tous été frappés, plusieurs fois fréquemment, et ont vu frapper leurs camarades. C'était le plus souvent de gifles qu'il s'agissait ; mais encore cette correction était-elle assez forte pour qu'une fois le jeune Brocard, ayant baissé la tête, ait reçu sur les doigts le poing de M. Frégnac avec une violence telle, que la main a été abîmée et qu'un ongle est tombé.

Les gifles ne constituaient pas, d'ailleurs, la seule punition corporelle ; un grand nombre d'enfants a reçu des coups de baguette sur les mains, surtout sur la tête.

Des coups de pied ont également été donnés ; des renseignements pris, il résulte que cette correction accompagnait d'ordinaire la mise à la porte de la classe ${ }^{13}$.

En mars 1889, l'inspection de l'administration générale de l'Assistance publique envoie au directeur de l'Enseignement primaire de la Seine un rapport sur deux instituteurs détachés à l'Hospice des Enfants assistés, rue d'Enfert :

J'ai abordé la question de front et ces messieurs m'ont avoué le mode de faire suivi par eux. 
Voici leurs déclarations :

M. Gérard avoue qu'il lui est arrivé de donner des coups de règle aux enfants une dizaine de fois, peut-être une vingtaine de fois en exagérant les choses, pendant sa carrière d'instituteur aux enfants assistés, et cela dans le creux de la main (M. Gérard est depuis 6 ans aux enfants assistés). Cette peine était bien méritée. C'était après un rappel à l'ordre de 50 fois au moins.

[...]

M. Petiteau déclare donner de temps en temps des coups de règle dans la main des enfants qui sont désobéissants. Ces coups de règle sont des coups de bâton à conduire les cerceaux.

Il ne peut préciser le nombre de fois qu'il s'est servi de la règle, il dit être des journées entières sans s'en servir, cependant, il en a donné ce matin à un nouvel arrivant.

J'ai interrogé six enfants au hasard, y compris le nouvel arrivant dont il est parlé ci-dessus, tous ont reçu des coups de règle. [...]

Le voisin du $2^{\mathrm{e}}$ et $\mathrm{du} 5^{\mathrm{e}}$ a reçu un coup de règle, le nouvel arrivant depuis lundi matin, c'est-à-dire depuis quatre jours, a reçu sept coups de règle.

Les coups donnés dans la main sont assez forts pour faire pleurer les enfants. Quand ceux-ci ne pleurent pas, c'est parce qu'ils se retiennent par amour-propre (déclaration des enfants), tous les jours le maître donne des coups de règle, non seulement dans la main, mais sur les jambes, dans le dos et quelquefois sur la tête ${ }^{14}$.

Les faits reprochés aux instituteurs sont généralement caractérisés par l'existence de traces visibles ou de séquelles plus graves. Ainsi, en 1884, l'inspecteur primaire dont dépend l'école de la rue Lavieuville informe le directeur de l'Enseignement primaire que Jules Boulay a frappé un élève de son cours élémentaire.

Trouvant que l'enfant n'obéissait pas assez vite, il le prit par l'oreille et lui donna un soufflet. L'ongle du doigt laissa sa trace sur le pavillon de l'oreille et la traction produisit, au point de séparation de l'oreille, quelques petites plaies linéaires. Le lendemain, le pavillon de l'oreille se trouva entièrement gonflé et le gonflement gagna le cou et la joue ${ }^{15}$.

Le 19 avril 1892, M. Dariot, directeur de l'école de l'impasse des Provençaux avertit l'inspecteur que

Mme Sangay m’a amené son fils, élève de la classe de M. Brunot. Il y a 8 jours, cet enfant a reçu de son maître, dit la mère, un coup de pied dans la cuisse. J'ai vu la blessure ; c'est encore tout noir. 
De la même façon, dans son rapport du 27 avril 1892, l'inspecteur note : Je ne pouvais faire utilement une enquête avant la rentrée des maîtres et des élèves. Je l'ai faite hier. J'ai d'abord entendu l'élève plaignant, le jeune Sangay Auguste, âgé de 10 ans. Il m'a déclaré que le 13 avril dernier, M. Brunot lui ayant demandé de répéter l'énoncé d'un problème donné, il n'avait pu le faire, ne s'en souvenant pas. Pour cette simple défaillance de mémoire, il reçut de M. Brunot un violent coup de pied qui l'atteignit à la cuisse gauche. J'ai vu les traces du coup, très apparentes encore après treize jours ${ }^{16}$.

En octobre 1880, Auguste Baudrier, adjoint à l'école de la rue du Renard, passe, quant à lui, littéralement un élève à tabac :

Le jeune Louis Arnaud est un petit employé du Bulletin des Halles, âgé de 13 ans et demi, ancien élève des frères de la rue des Blancs-Manteaux, qui suivait le soir la première classe du cours d'apprentis de la rue du Renard. Il n'est que trop constant que les soufflets qu'il a reçus du maître, M. Baudrier, pour n'avoir pas écouté un problème fait au tableau, et n'avoir pu le reproduire, ont occasionné une enflure de l'œil et de la joue, avec épanchement intérieur de sang et coloration bleue de l'orbite, assez visible le lendemain pour que le signataire de la lettre [secrétaire de la commission de protection des enfants en bas âge du $3^{\mathrm{e}}$ arrondissement], rencontrant l'enfant et croyant qu'il s'était battu, l'interrogeât sur l'origine des coups ${ }^{17}$.

Le 28 mai 1888, le maire d'Aubervilliers signale à l'inspecteur primaire de la circonscription, M. Brouet, qu'un élève de l'école des Quatre-Chemins aurait été frappé « avec une telle violence que l'enfant aurait été obligé de garder le lit pendant plusieurs jours et conserverait des traces des coups reçus". Dans son rapport, M. Brouet constate que

le jeune Courteaux a été appréhendé par M. Bayle, le 22 mai, et frappé à coup de règle sur différentes parties du corps, notamment aux cuisses et au dos. Rentré chez lui, l'enfant se mit en mesure de faire ses devoirs, mais se tint debout. La mère, surprise de cette attitude, lui en demanda les motifs, et l'enfant lui répondit qu'il avait été battu en classe, et qu'il ne pouvait s'asseoir. Elle l'examina et remarqua de fortes ecchymoses dans différentes parties du corps. [...] Je me suis rendu à Aubervilliers le 25 mai. J'ai visité l'enfant, et j'ai reconnu sur les cuisses des traces de coups évidentes ${ }^{18}$. 
Plusieurs dossiers font état de plaintes réparties tout au long de la carrière de l'instituteur. Par exemple, en novembre 1888, André Joret est accusé de punir les enfants en leur donnant des coups de baguettes et en leur interdisant de se rendre aux latrines, au point que certains ont uriné dans leurs vêtements. En janvier 1890, l'inspecteur Laurent note qu'" ...il est certain que M. Joret donne parfois des taloches à ses élèves ". Une jeune élève " a reçu sur le nez une légère claque qui a déterminé une perte de sang ". Au mois de mars 1891, l'inspecteur Berthon indique que "M. Joret semblait vouloir faire oublier le passé ; il a fait des efforts dans ce sens... mais ses bonnes résolutions n'ont pas persisté : il s'est laissé aller à frapper des élèves " ${ }^{19}$. Pierre Aubry est un bon exemple de ces instituteurs particulièrement violents qui font un usage fréquent de la brutalité et que les plaintes ne parviennent pas à arrêter. Le 5 septembre 1883, le directeur de l'école de la rue de la Motte-Picquet détaille, dans une lettre au directeur de l'Enseignement primaire, les " coups et blessures " dont il accuse un de ses adjoints, qui s'en prend à l'ensemble des élèves de l'école. L'inspecteur fournit une liste apparemment non exhaustive de 12 élèves "qui ont été le plus souvent frappés (du poing ou du pied, quand ce n'est pas du bâton) par M. Aubry ".

Une mention particulière doit être faite pour l'affaire Rollin (Achille), $2^{\mathrm{e}}$ cours supérieur : M. Aubry est allé le chercher à la classe à l'étage au dessus de la sienne, l'a descendu en $3^{\text {e }}$, l'a fait monter sur 2 escabeaux superposés et l'a tarabusté jusqu'à ce que l'élève, perdant l'équilibre, ait entraîné dans sa chute l'entourage du poêle auquel il cherchait à se retenir et qui est tombé sur M. Aubry entrainé dans sa chute en présence de tous les élèves de la $3^{\mathrm{e}}$.

Guant à l'élève Madrias, le fait qui le concerne est plus grave encore et le voici : l'élève appuyait sa main sur le rebord de la table, M. Aubry voulant le faire cesser cherche à lui donner un coup de règle carrée sur le dos de la main, l'enfant retire brusquement la main, la règle se brise sur la table et rebondit sous la paupière inférieure qu'elle traverse pour atteindre l'œil même.

Quelques années plus tard, en juin 1889, le directeur de l'école de la rue de Pontoise rapporte que, comme il pleuvait, les élèves ayant fini de déjeuner, " M. Aubry avait fait faire à ses élèves le tour du préau. Pendant cet exercice, le jeune Gibert, s'étant écarté de son rang, fut rappelé à l'ordre par le maître qui, de plus, lui donna quelques petits coups secs avec la canne dont il se servait pour marquer le pas. L'élève ainsi frappé prononça des paroles impolies ; M. Aubry, irrité, lui donna un soufflet ". Un mois plus tard, de nouvelles plaintes se pro- 
duisent. Un médecin atteste que l'élève Duclos a sur l'épaule " une ecchymose large de deux centimètres produite par un coup de canne ", alors que le père de l'élève Lefebvre affirme qu'il " est revenu samedi soir avec un coup de canne sur la tête parfaitement marqué ; du reste la noirceur existe encore et en le faisant appeler vous pourrez vous rendre compte ". Pourtant, de tels actes de violence, s'inscrivant sur le corps des élèves, semblent, par leur répétition, tolérés dans une certaine mesure.

\section{II - Une violence partiellement justifiée par les contemporains}

Quand la réaction des instituteurs aux accusations dont ils sont l'objet est connue, la plupart d'entre eux semblent admettre les faits et reconnaître leurs torts. Trois seulement nient partiellement, malgré le démenti des témoins, les inspecteurs s'efforçant de recueillir les témoignages des élèves et des collègues de l'instituteur incriminé. Ainsi, Pierre Aubry

a prétendu que, depuis le 11 mai [1883], il n’avait infligé aucun châtiment corporel. Or, sur ce point, les élèves Vial, Voisin, Sotinel et Cornet lui donnent un démenti formel. Lecomte et Barzie ont même été bousculés depuis la rentrée ${ }^{20}$.

De la même façon, à propos de l'attitude de Marie Peretti, l'inspecteur remarque qu'" elle a en outre le grave défaut de nier contre toute évidence. C'est chez elle un système qu'elle soutient avec une véritable effronterie $"^{21}$.

L'attitude la plus courante est cependant l'expression de regrets, qui apparaissent dans huit cas parmi les onze dossiers dans lesquels l'inspecteur fait état des sentiments des instituteurs. Deux d'entre eux paraissent particulièrement affectés par les plaintes. En avril 1892, Paul Brunot est désemparé face aux accusations.

M. Brunot, qui a entendu toutes ces accusations, en est atterré. Les enfants renvoyés, je lui fais remarquer que les témoignages sont trop concordants pour ne pas être sincères. Il ne nie pas, mais il dit ne pas se souvenir ; il assure qu'il est plein de bienveillance pour Sangay qui est une bonne nature; il ne comprend rien à ce qui est arrivé, si c'est arrivé. 
En mai 1886, Auguste Scellos, ayant bousculé un élève qui s'est gravement blessé à la tête dans sa chute, est effondré.

Au fond, M. Scellos est plus malheureux que coupable. Il n'y a pas eu intention de sévir contre l'enfant. Le mal est que M. Scellos ne s'est pas rendu compte de l'effort de son mouvement imprudent sur la personne d'un faible enfant. Il déplore son erreur ; son abattement fait mal et je suis certain qu'il a déjà payé sa faute par les cruelles souffrances morales qu'il a endurées.

La crainte des sanctions joue un rôle dans ce repentir, par exemple quand Jacques Laboureau se met à pleurer en demandant à l'inspecteur, qui le menace, de prendre en pitié sa situation de père de famille ${ }^{22}$. Il est donc difficile de saisir les conceptions éducatives qui motivent le recours à des punitions corporelles, même si certains cas permettent d'appréhender les représentations de l'usage légitime de la violence qui animent ces instituteurs. François Cartier continue de frapper les élèves de la main en présence de l'inspecteur. Gérard et Petiteau, instituteurs à l'Hospice des Enfants assistés, minimisent la gravité de leurs actes.

M. Gérard, revenant aux coups de règle, dit que ce genre de punition, auquel il n’a eu recours que dans des circonstances rares, était plutôt pour obtenir chez les enfants un effet moral qu'un résultat physique. Les enfants ayant plus de 11 ans, il pensait qu'ils étaient humiliés de venir recevoir cette correction qui ne pouvait leur faire aucun mal et que du reste il ne les donnait pas de façon à leur faire du mal.

M. Petiteau déclare donner de temps en temps des coups de règle dans la main des enfants qui sont désobéissants. [...] Quelquefois il lui est arrivé de faire mettre les enfants à genoux, peu de temps, dit-il ; il dit ne pas avoir d'autres moyens de répression, il considère cela comme une correction anodine, il se garderait bien de maltraiter les enfants, il n'a jamais tiré les oreilles de ceux-ci.

Le témoignage du surveillant chargé de ces enfants en dehors de la classe confirme que l'usage de punitions corporelles est alors largement toléré dans cette institution. En effet, quinze jours environ avant les faits, il a été, par hasard, témoin de coups de règle infligés à un élève, ce qui n’a entraîné de sa part aucune réaction.

Seule la douleur physique est prise en compte dans ces déclarations. Le fait que cette violence soit exercée par une autorité bénéficiant d'une forte légitimité sociale et le traumatisme psychologique constitué par la volonté d'humilier sont implicitement niés. En outre, certaines affaires suggèrent que la violence de ces 
instituteurs suscite une enquête en raison du caractère excessif de cette violence plus que du seul fait de son usage. Certains plaignants font preuve d'une grande tolérance. Ainsi, un parent d'élève, M. Deschamp, déclare en novembre 1889 : " Je me suis rendu près de Monsieur le Directeur de l'école, qui m'avait promis de sermonner le maitre ${ }^{23}$. Quant à M. Sailly, père d'un élève de l'école de la rue Geoffroy l'Asnier, il explique, en novembre 1891, qu'il a déjà menacé de porter plainte en raison des mauvais traitements dont avait été victime son enfant à trois reprises en mai-juin, mais qu'il " en fut dissuadé sur l'assurance qu'on lui fit que cela ne se renouvellerait plus ". C'est la troisième récidive de l'instituteur qui entraîne une plainte officielle ${ }^{24}$. Pendant la même période, une plainte anonyme dénonce les brutalités dont Edgard Merlin, instituteur à Saint-Denis, se serait rendu coupable. L'inspecteur constate que les coups de règle carrée que le jeune Pailloux a reçus ont laissé des traces bien visibles sur ses fesses, mais que la mère avait demandé au directeur de l'école une réprimande sévère pour le maître sans envisager une plainte à l'administration. L'inspecteur ajoute d'ailleurs que "les parents n'ont pas l'intention d'attirer de désagrément à l'instituteur " ${ }^{25}$. Dans le cas de l'élève des cours du soir pour apprentis de la rue du Renard durement frappé au visage par Auguste Baudrier, sa grand-mère maternelle refuse de porter plainte malgré l'insistance du secrétaire de la commission de protection des enfants en bas âge du $3^{\mathrm{e}}$ arrondissement, qui a repéré l'adolescent dans la rue. Elle explique sa décision en déclarant " ne pas vouloir faire perdre sa place à un maitre ". Cette acceptation d'une violence punitive considérée comme légitime apparait aussi dans le cas de Paul Brunot, accusé d'avoir frappé le fils de $\mathrm{M}^{\mathrm{me}}$ Meunier. Celle-ci avoue à l'inspecteur que " son mari corrigeait parfois ses enfants et que les coups dont se plaignait son fils pourraient bien venir de lui ". Guand Pierre Aubry blesse un élève à l'œil en 1883 et que la mère de l'enfant vient se plaindre, il « va s'excuser auprès du père, et sort triomphant de chez M. Madrias, annonçant bruyamment partout que, loin de lui faire des reproches, M. Madrias lui a payé une bonne bouteille, d'où scandale en famille".

Cet exemple souligne le caractère sexué de ces formes très brutales de punitions corporelles et le partage du droit de correction paternelle entre le père et l'instituteur. De plus, il exprime d'autant mieux la relative banalité de l'utilisation des châtiments corporels que les élèves eux-mêmes ont intériorisé la légitimité de

23 D1T1 41, dossier Joret.

24 D1T1 305, dossier Duchenne.

25 D1T1 252. 
ces procédés. Devant l'inspecteur, qui les interroge en particulier dans le cabinet du directeur de l'école, tous les élèves concernés " ont reconnu d'ailleurs que leur conduite avait amené ces corrections, et ils ne semblent pas, en général, garder rancune à M. Aubry ". Cette attitude peut être attribuée en partie au respect de l'institution scolaire et de l'autorité, très valorisée par l'école de cette époque. Toutefois, quand l'inspecteur primaire du $18^{\mathrm{e}}$ arrondissement, M. Daix, constate que M. Laurent, instituteur à l'école de la rue de Clignancourt a tendance à donner "quelques claques de temps à autres ", il se hâte d'ajouter que "le maître est aimé de tous ses élèves sans exception. L'un d'entre eux me dit lui-même, avec une entière franchise, avoir reçu quelques coups de règle bien mérités " ${ }^{26}$.

Enfin, dans le cas de Clovis Frégnac, autre instituteur particulièrement brutal, "les enfants ont été très affirmatifs sur les coups qu'ils ont reçus et vu donner, ce qu'ils racontent d'ailleurs sans récriminer, reconnaissant pour la plupart "qu'ils l'avaient bien mérité" ".

\section{III - Les conditions du recours à la violence}

Cette relative tolérance envers les punitions corporelles employées par les instituteurs peut aussi s'expliquer par les circonstances dans lesquelles les faits se sont produits, par la fragilité de la position de l'instituteur et, parfois, par la responsabilité de l'institution scolaire elle-même. En effet, les documents font peu état de véritables pulsions sadiques. Le seul exemple identifié comme un acte de brutalité gratuit, motivé uniquement par l'assouvissement de telles pulsions, apparaît dans la plainte d'un parent d'élève.

Mon fils, âgé de 11 ans, va à l'école communale de la rue de l'Ouest dirigée par Monsieur Lavallée depuis lâge de 6 ans. Il fait partie de la $2^{\mathrm{e}}$ classe et il a été frappé violemment par un maître de la 5 e. Pendant la récréation, étant assis sur un banc, ce maitre passe devant lui en lui disant : "il y a longtemps que je ne t'ai pas battu " et en même temps il le gifle à plusieurs reprises à tel point que les marques en sont restées empreintes pendant quatre jours ${ }^{27}$.

Évidemment, ce type de pulsion n'est pas forcément absent des autres dossiers, surtout parmi ceux dans lesquels les actes de brutalité apparaissent comme une pratique quotidienne. Mais les documents ne permettent pas d'accéder à une perception, même superficielle, du psychisme des individus concernés. De plus, 
dans les rapports des inspecteurs, les faits sont dans l'ensemble décrits comme des actes de violence soudains, conséquences de l'exaspération des instituteurs. Les inspecteurs et les directeurs d'école expliquent parfois, sans les excuser, les faits reprochés aux instituteurs dans ce type de dossier par les difficultés personnelles qu'ils rencontrent pour maintenir la discipline ; ils reconnaissent même une part de responsabilité de l'institution, notamment quand les conditions d'enseignement sont trop mauvaises en terme d'effectifs et de type de public scolaire.

En premier lieu, les inspecteurs font souvent état de l'indiscipline des élèves pour expliquer qu'un instituteur se soit laissé aller à frapper un élève. Dans neuf dossiers sur quarante, les inspecteurs considèrent les actes de brutalité comme des dérapages explicables par l'exaspération légitime des instituteurs. En juillet 1899, Hélène Créplot donne deux soufflets à une élève, rappelée à l'ordre pendant le déjeuner, qui répond à l'institutrice en l'accusant de mentir. L'inspecteur précise que "l'enfant est une élève très indocile, impolie d'habitude avec tout le monde ".

À propos d'Albert Duchenne, l'inspecteur Chaumeuil note que " ce maître très distingué, froid et calme, n'a pas l'habitude de maltraiter ses élèves, mais il a été poussé plusieurs fois à bout par l'indiscipline et le caractère frondeur du jeune Sailly".

L'inspecteur Daix présente un cas similaire :

Depuis 11 ans que M. Audiard est à l'école de la rue Tandou, il n'est parvenu aucune plainte sur son compte. Quant au jeune Huret, qui faisait partie de l'école de la place de Bitche, avant les vacances, il avait déjà créé des difficultés au directeur de cette école, qui en ce moment est enchanté de ne plus l'avoir.

Dans sept dossiers, les grandes difficultés que rencontrent les enseignants pour établir la discipline collective sont avancées pour expliquer que les instituteurs, débordés par leurs élèves, en arrivent à user de violences physiques, aveu d'impuissance face à cette situation.

Les redoublants sont au nombre de 56 ; ils viennent de toutes les écoles du quartier ; ce sont au début de l'année les élèves les plus difficiles à conduire. Le maître est nouveau pour eux, et si je puis m'exprimer ainsi, ils ne manquent pas de le tâter. L'ordre est surtout malaisé à établir dans la montée et la descente des escaliers. Il faut donc une grande dose de patience pour ne pas se laisser aller à certaines vivacités. M. Audiard Paul n’a pas su, malheureusement, se maîtriser assez ${ }^{28}$. 
La situation de M. James devient de plus en plus intolérable. À certains moments, sa classe est si bruyante que, dans la salle contiguë, le maître de la $6^{\mathrm{e}}$ classe ne peut continuer son travail. Les élèves se roulent par terre, se donnent des coups, se griffent, montent sur les bancs, se mettent même à genoux sur les tables, se jettent de l'encre ; j'ai reçu des plaintes de parents à propos de ces coups et de l'encre.

Comme il est incapable de faire un service de préau ou de déjeuner, il surveille la récréation. Là encore, sa présence provoque le désordre. Il faut que les élèves me voient avec lui. Si je m'écarte, les enfants jouent dans les cabinets, grimpent après les supports de la toiture allant aux cabinets ou sur les cloisons des urinoirs.

Hier, il s'est produit un incident assez sérieux. Quelques élèves se mirent à chanter : "Voilà du bon fromage au lait, il est du pays de celui qui l'a fait ". M. James, voulant les faire taire, se précipite sur un élève, celui-ci se sauve, le maître le poursuit ; vous pensez comme cette chasse amusait les enfants. Le fugitif, rejoint, eut les joues tortillées et fut mis au piquet. Pendant ce temps, un autre disait à son voisin : "Comme il est méchant M. James ". - " Attrapez-moi celui-là ", s'écrie le maître. Un coup de poing dans le dos, un sur le nez en punition. L'enfant a saigné abondamment... ${ }^{29}$.

Les inspecteurs, qui connaissent très bien la géographie sociale du réseau scolaire de Paris et de sa banlieue, savent évaluer les difficultés spécifiques que pose le maintien de la discipline dans certaines écoles importantes de la périphérie en cours d'urbanisation. Ainsi, la moitié des cas étudiés se situent dans les écoles des quartiers ouvriers périphériques $\left(18^{\mathrm{e}}, 19^{\mathrm{e}}\right.$ et $20^{\mathrm{e}}$ arrondissements, auxquels il faut ajouter le $13^{\mathrm{e}}$ ) et des communes de banlieue limitrophes. Les inspecteurs savent que les élèves de ces écoles se soumettent moins facilement à la discipline scolaire $^{30}$. Par exemple, en novembre 1889, l'inspecteur Laurent explique la brutalité d'André Joret par la conjonction de la rigidité de sa discipline toute militaire et du public scolaire populaire et turbulent de l'école de la rue Damrémont (18 ${ }^{\mathrm{e}}$ arrondissement).

M. Joret est chargé d'une division du cours élémentaire ; les enfants de ce quartier sont souvent insoumis et insolents, et les jeunes, comme les plus âgés, ne sont pas faciles à diriger. Cette situation peut expliquer le mécontentement ou l'impatience du maître, mais non justifier ses actes de brutalité.

\section{D1T1 230.}

30 On notera toutefois que les dossiers ne font jamais état d'actes de violence des élèves dirigés contre le maître, de nature à entraîner un renvoi immédiat de l'élève ; tout au plus quelques bagarres en cour de récréation et quelques chahuts contre des maîtres manquant d'autorité sont signalés. 
M. Joret est un ancien militaire. Il est très sévère. Son allure, son maintien, sa voix annonce un tempérament dur et hautain. Il n'est pas étonnant que ce maître ne gagne pas l'affection de ses élèves ni la sympathie de son directeur.

Dans quatre cas particulièrement importants par l'ampleur des plaintes et leur gravité, les inspecteurs développent une analyse approfondie des dysfonctionnements qui ont facilité soit un passage à l'acte aussi brutal qu'inattendu, soit l'usage quotidien de punitions corporelles. L'inspecteur Drouard en vient ainsi à plaider les circonstances atténuantes pour les deux instituteurs de l'Hospice des Enfants assistés de la rue Denfert, tout en condamnant les punitions corporelles employées.

Je crois devoir vous rappeler, M. le Directeur, que l'Hospice des Enfants assistés [...] reçoit les enfants que l'Assistance publique vient de prendre à sa charge et qu'il ne les garde que pendant une courte période dite d'observation (quelques jours, un mois, rarement 3 mois), après laquelle les enfants sont dirigés sur les établissements ou sur les agences qui doivent les recevoir définitivement.

Les garçons âgés de plus de 7 ans sont soumis, pendant leur séjour dans l'établissement, à un enseignement général donné par les deux instituteurs sur lesquels on a appelé notre attention et à des exercices manuels dirigés par des ouvriers (menuiserie, sonnerie et confection de chaussons). Il y en a de tous les âges (6 à 20 ans) et de degrés d'instruction très variables. Les plus âgés sont sous la direction de M. Gérard ; les plus jeunes sont instruits par M. Petiteau. L'effectif des deux classes est en moyenne de 120 élèves. Cet effectif se renouvelle presque entièrement chaque mois, puisque près de 2000 garçons passent annuellement entre les mains des instituteurs.

Dans ces conditions, les maîtres ne peuvent pas donner un enseignement régulier ; ils occupent les enfants par des exercices écrits correspondant à la force de chacun d'eux ; ils cherchent à les intéresser par des leçons orales et par des lectures. Ils ne peuvent faire ni plus ni mieux.

Vous remarquerez qu'une telle tâche est ingrate, difficile. Les maîtres ont affaire, en général, à des enfants dont l'éducation a été mal dirigée ou négligée ; ils ne peuvent pas, comme les instituteurs chargés d'une classe régulière, agir sur le caractère de leurs élèves par l'enseignement et un système rationnel de punitions et de récompenses. Il faut pourtant qu'ils obtiennent de l'ordre dans la classe. Aussi, tout en reconnaissant qu'il leur est interdit pour cela d'employer des châtiments corporels, je m'explique parfaitement que la patience leur fasse quelquefois défaut et qu'ils se laissent aller à l'usage de procédés coercitifs regrettables, regrettables parce que l'instituteur, en les employant, perd de sa dignité, parce qu'ils aigrissent ou révoltent l'esprit des enfants et qu'ils vont ainsi à l'encontre du but qu'on se propose. 
J'ajoute que les deux maîtres, détachés du service général, sont à peu près sans direction pédagogique : ils ne sont ni inspectés, ni conseillés comme le sont leurs collègues des autres écoles publiques.

Le cas de cet établissement particulier ne peut être généralisé mais, dans deux autres dossiers, la situation de crise que connaît une école, aggravée par le contexte politique tendu sur la question de la laïcité, accroît ses difficultés, crée les tensions qui aboutissent au passage à l'acte de l'instituteur. En novembre 1880, quand Auguste Baudrier frappe le jeune Louis Arnaud, l'école de la rue du Renard n'est plus vraiment dirigée. Elle n'a pas été laïcisée et le directeur, M. Grenier, a peu d'autorité et est confronté depuis le printemps à l'opposition de ses adjoints qui, anticipant le vote de la loi Ferry laïcisant les écoles publiques, refusent d'accompagner les élèves au catéchisme le jeudi, aux préparatifs de la première communion et aux offices les dimanche et jours de fêtes. L'école est par ailleurs soumise à une forte concurrence scolaire " en présence d'une nouvelle école des Frères, fondée par le curé de Saint-Merry, rue de la Verrerie, et devenue très peuplée sans qu'il y ait eu laïcisation d'école communale ". Le directeur se heurte particulièrement à Baudrier, jeune instituteur républicain sorti de l'école normale de la Seine en 1875, dont le déchaînement de violence contre le jeune Louis Arnaud, âgé de 13 ans et demi et " ancien élève des Frères de la rue des Blancs-Manteaux ", ne peut s'expliquer seulement par son manque d'attention lors de la correction d'un problème. D'ailleurs, l'inspecteur Dupaigne insiste sur la responsabilité du directeur de l'école :

Mais s'il y a lieu de se plaindre de la violence du maître, il n'y a pas moins lieu de se plaindre de la faiblesse du directeur de l'école, M. Grenier, qui, attiré par le bruit, a été témoin des derniers coups donnés par le maître, sur cette seule protestation de l'enfant : "vous n'avez pas le droit de me frapper ", et qui n'a rien trouvé à dire que de faire sortir l'enfant, le faire taire et l'immobiliser dans un coin sans regarder s'il était blessé ou non, sans témoigner un mot de blâme pour l'impatience et la violence du maître... Le seul reproche fait au maître pris en flagrant délit, qu'il eût dû immédiatement remplacer en classe et envoyer se calmer dans son bureau, puis conduire avec l'enfant chez la mère pour faire des excuses, consiste en une timide allusion à la page 3 d'une circulaire envoyée aux maîtres quatre jours après l'affaire, et où il se plaint surtout du tort personnel que fait à ses loisirs l'inexactitude des maîtres au service du dimanche.

En 1905, Édouard Madeuf, le directeur de l'école de la rue de Passy, déclare que les punitions corporelles ne sont pas en usage dans son école mais que "quelques 
actes d'impatience, qu'il ne faut point amplifier, ont été commis ". Il les explique, et l'inspecteur lui donne raison, par la croissance démographique des arrondissements périphériques et la précarité de l'installation matérielle de l'école, aggravées par les origines sociales et culturelles des élèves et la fermeture de certains établissements congréganistes en conséquence de la loi du 7 juillet 1904.

Sur les huit classes de l'école, quatre sont normales, deux sont établies dans d'anciens appartements et les deux autres sont installées dans le préau ; il en résulte un encombrement qui énerve les élèves et encore plus les maîtres. Les récréations qui devraient procurer un moment de repos sont au contraire une cause de préoccupations plus grandes : les enfants n'ont pas chacun un mètre carré pour jouer ; les chutes sont nombreuses et journalières ; si un enfant tombe, un autre le piétine ; il en résulte des écorchures et des blessures dont plusieurs auraient pu être graves (dents cassées, nez écrasé, etc.). [...]

En outre, l'augmentation de la population du quartier a introduit dans l'école un élément de trouble et de désordre : ce sont des enfants renvoyés des écoles congréganistes ou qui appartiennent à une colonie italienne ; d'autres forment un groupe hétérogène par l'origine, mais très homogène par les habitudes de vagabondage.

Contre ces enfants, que faire ? Les punitions réglementaires (mauvaises notes et retenues) sont sans effet : l'exclusion serait un moyen, non de les améliorer, mais de les empêcher de troubler les autres ; seulement, ces élèves vont rarement jusqu'aux cas graves et extrêmes qui la peuvent motiver ; et puis la situation générale ne serait pas modifiée par la suppression de deux ou trois unités sur une trentaine.

On conduira ces enfants au directeur ; celui-ci n'est pas plus armé que les adjoints, et son intervention n'aura d'effet que si elle est exceptionnelle.

[...]

Ainsi, qu'on me pardonne un langage plus franc qu'administratif, mais ces mouvements d'impatience blâmables au point de vue réglementaire, sont souvent excusables si l'on considère l'instituteur.

[...] Si l'instituteur s'impatiente, parce qu'il prend sa tâche à cœur et parce que, malgré sa surveillance et son activité, certains élèves indociles troublent constamment l'ordre, empêchent le travail et narguent le maître, je ne puis m'empêcher de l'excuser. C'est le cas qui a été relevé à l'école. ${ }^{31}$

Dans ce cas, l'entassement des élèves dans des locaux inadaptés et l'hétérogénéité du public scolaire favorisent l'exaspération des maîtres et le désarroi d'un directeur désarmé face à cette situation. La difficulté à scolariser une population d'extraction modeste, parfois culturellement défavorisée, le faible encadrement 
dans des établissements regroupant plusieurs centaines d'élèves et le sentiment d'impuissance à imposer un ordre scolaire qui n'est pas reconnu par l'environnement social sont alors considérés comme des facteurs déclencheurs de la violence des maitres. Toutefois, lorsque les faits relèvent pleinement de la punition corporelle, les plaintes témoignent de la progression du refus de ces violences.

\section{IV - Un refus croissant de la violence}

Si la relative tolérance de la société environnante vis-à-vis des punitions corporelles peut favoriser le passage à l'acte et si le contexte matériel, social et politique suscite des tensions génératrices de violences, il n'en reste pas moins que l'analyse des dossiers témoigne chez les plaignants d'un refus le plus souvent catégorique de l'usage de telles punitions. Quand un enseignant est connu pour sa brutalité quotidienne avec les enfants, il arrive que certains parents décident de le changer d'école ou s'adressent au directeur de l'établissement pour demander un changement de classe, l'organisation concentrique des programmes facilitant probablement ce type de stratégie. À l'école de la rue de Lourcine, en octobre 1886, la directrice indique à l'inspecteur que

la brutalité de $\mathrm{M}^{\mathrm{me}}$ Barthel inspire une telle crainte aux enfants que beaucoup de parents me demandent de ne pas faire passer leur fille dans cette classe, préférant la retirer de l'école plutôt que de la voir confiée à $\mathrm{M}^{\mathrm{me}}$ Barthel.

Malgré mes nombreuses observations, $\mathrm{M}^{\mathrm{me}}$ Barthel traite ses élèves avec une telle dureté que chaque jour les parents viennent me prier de ne point laisser leur fille dans sa classe ; en sorte que je me vois forcée ou de mettre des élèves trop faibles dans la première division du cours moyen, ou de laisser des élèves trop fortes dans le cours élémentaire ; lundi dernier encore, $\mathrm{M}^{\mathrm{me}}$ Lévy s'est plainte que sa fille avait été brutalisée à plusieurs reprises, et m’a prévenue qu'elle allait la reprendre si je la laissais entre les mains de $\mathrm{M}^{\mathrm{me}}$ Barthel.

Les parents insistent beaucoup sur le fait qu'ils acceptent que leur enfant soit puni, même sévèrement, s'il a fait preuve d'indiscipline, mais qu'ils refusent toute punition corporelle. Ainsi, cette mère d'élève porte plainte contre Marie Barthel en précisant :

Si l'enfant n'écoute pas, punissez-la à midi, en l'envoyant chercher son déjeuner et je me charge de lui donner du pain sec, et ne la laissez pas sortir à la récréation, mais je ne veux pas que l'on la frappe de n'importe quelle façon et pas de manière brutale. Depuis un an avec $\mathrm{M}^{\text {elle }}$ Emma je n'ai jamais eu à m'en plaindre, je la 
trouvais plutôt trop pacifique mais cette fois ce sont les deux extrêmes : l'une trop bonne, l'autre trop dure, mais je préfère la première car, dans le métier que cette personne a entrepris, il faut de la douceur, du calme et de la persévérance, car ce sont des enfants qu'elle dirige ${ }^{32}$.

Pour M. Sailly, parent d'élève de l'école de la rue Geoffroy l'Asnier en 1891, quelle que soit la désobéissance ou l'espièglerie de l'élève, il est à la portée du maître d'autres punitions que les coups, souvent dangereux et toujours immoraux.

Quand les punitions corporelles sont plus graves et que les enfants concernés en portent des marques visibles, le ton des plaintes change et devient naturellement plus agressif. Et ce malgré l'intervention du directeur de l'école, qui tente de temporiser, en tant que représentant de l'institution scolaire et premier interlocuteur des parents qui viennent protester à l'école avant la rédaction d'une lettre officialisant la plainte. Les parents d'élèves opposent souvent l'instituteur bourreau et les enfants martyrs, comme dans le cas de Clovis Frégnac, directeur de l'école de Villemomble en 1889. Alors que l'orthographe et la syntaxe de leurs écrits montrent qu'ils sont issus d'un milieu culturellement favorisé, deux parents d'élèves, MM. Roger et Lepère, usent du vocabulaire de la citoyenneté pour formuler leur plainte, dans le contexte du centenaire de la Révolution française où la jeune République exalte l'accomplissement de l'idéal révolutionnaire.

Les parents qui, d'après la nouvelle loi sur l'enseignement, sont forcés d'envoyer leurs enfants à l'école, ne sont pas obligés de les confier à des bourreaux. [...] Et cela se passe au su de l'inspecteur dans une commune du premier département de France et en pleine République ! [...] L’État, qui impose les plus grands sacrifices pour donner une bonne instruction à notre jeune génération, a toujours, et de tout temps, défendu aux maîtres de brutaliser les élèves confiés à leurs soins, ni de devenir un objet de vexation pour aucun citoyen.

$[\ldots]$

Par conséquent, je demande donc à M. le Préfet, au nom des parents de Villemomble, au nom des enfants martyrs, au nom de cette génération future sur laquelle tant d'espoir est fondé, de vouloir faire rétablir immédiatement les récréations de la journée et d'ordonner le plus tôt qu'il sera possible le changement de cet ignoble et féroce instituteur.

[...] Nos enfants ne peuvent dans de pareilles conditions avoir la liberté d'esprit nécessaire pour que les leçons puissent leur profiter ; ils sont tous sous l'empire 
d'une crainte de tous les instants qui les subjugue complètement. Et dire que nous sommes dans un pays libre ; quelle belle éducation ? La brutalité, les coups, le knout employés dans une école française du département de la Seine.

Ce refus des punitions corporelles ne se limite pas aux parents et peut être parfois le fait des élèves, individuellement ou collectivement, quand ils sont assez âgés pour le faire. Quand Auguste Baudrier frappe le jeune apprenti des cours du soir de la rue du Renard, celui-ci lui crie qu'il n'a pas le droit de le frapper. À l'Hospice des Enfants assistés, le jeune Boyer, élève assez insolent, âgé de 12 ans, aurait déclaré à l'instituteur Gérard, qui lui tirait l'oreille : " vous n'avez pas le droit de me toucher, je vais aller trouver M. le Directeur ", avant d'être exclu de la classe. Le lendemain, cet élève profite de son passage devant la commission centrale de surveillance du service des enfants moralement abandonnés, qui doit décider à la fin de leur période d'observation s'ils peuvent être admis définitivement, pour dénoncer les coups qu'il a reçus. Il déclenche ainsi une enquête après que d'autres élèves ont confirmé les faits. Ainsi, si l'usage de punitions corporelles reste très fréquent à cette époque, l'institution scolaire doit faire face à un refus croissant des abus que peuvent commettre une minorité d'instituteurs, comme le montre notamment l'absence, dans les dossiers, de plaintes pour punition corporelle antérieure à $1880^{33}$.

\section{V - Des pratiques sanctionnées}

L'institution scolaire ne peut laisser sans réponse des plaintes qui dénoncent des faits constituant une négation des principes dont elle se proclame l'incarnation. Les inspecteurs partagent l'indignation des plaignants face à des actes d'une grande brutalité et signalent souvent les avertissements qu'ils avaient déjà adressés à l'instituteur coupable. En septembre 1884, l'inspecteur Delapierre souligne ainsi :

Tout d'abord, M. Brajon est coupable, très coupable même. Dans toutes nos conférences, dans toutes nos réunions, j'ai toujours saisi l'occasion qui m'était offerte pour signaler aux maîtres et aux maîtresses l'interdiction absolue des peines corporelles quelles qu'elles soient. M. Brajon a donc péché en connaissance de cause. Et puis,

33 Ce rejet croissant de la violence, très sensible à partir du début des années 1880, a une traduction législative avec le vote de la loi du 24 juillet 1889 sur la protection des enfants moralement abandonnés et maltraités, puis de la loi du 19 avril 1898 réprimant les violences faites aux enfants. Voir le $\mathrm{n}^{\circ} 2$ de la revue Le Temps de l'Histoire, Cent ans de répression des violences à enfants, 1999. 
frapper un enfant avec un bâton constitue un acte de barbarie qu'on a peine à se figurer par le temps d'éducation pédagogique qui court ${ }^{34}$.

En janvier 1893, l'inspecteur Drouard, insistant sur la difficulté à mettre fin à la pratique des punitions corporelles, demande dans son rapport que la peine de réprimande soit bien infligée à Alfred Debroise.

J'insiste d'autant plus pour cette punition que, depuis quelque temps, dans les écoles de garçons, malgré les recommandations que j'ai faites dans les conférences pédagogiques, il semblerait que les corrections manuelles fussent moins évitées que par le passé.

En juillet 1905, un autre inspecteur se sent tenu de signaler qu'il a encore récemment rappelé le règlement à ses instituteurs.

J'espère qu'il n'y aura plus aucune punition corporelle ni dans cette école, ni ailleurs. Je viens, à ce sujet, dans ma dernière conférence pédagogique, de faire de nouvelles observations et recommandations à tout mon personnel des trois arrondissements.

Ces interventions répétées témoignent de la difficulté des inspecteurs primaires à faire respecter les règlements en vigueur, ce qui rend d'autant plus nécessaire à leurs yeux la prise de sanctions. Par ailleurs, les plaignants attendent d'abord de l'institution scolaire qu'elle sanctionne les pratiques qu'ils dénoncent. Dans quatre cas seulement, les parents ont recours directement à la justice en s'adressant au commissaire de police de leur quartier. Un seul instituteur est vraiment inquiété : le 30 juillet 1884, Léon Brajon est convoqué au commissariat et inculpé de " coups volontaires sur la personne d'un des enfants confiés à ses soins ". Il est laissé en liberté, mais la procédure lancée contre lui a été transmise au parquet par le procureur de la République. Toutefois, celle-ci ne semble pas avoir abouti : le directeur de l'Enseignement primaire avait décidé d'attendre les suites judiciaires de l'affaire pour prendre des sanctions, or il n'est plus question de cette affaire dans le dossier de Brajon par la suite.

Inspecteurs primaires et directeurs d'école s'efforcent d'ailleurs de dissuader les parents d'élèves de porter plainte en justice, car ce serait une manifestation de l'incapacité de l'institution scolaire à faire respecter elle-même ses principes et règlements. Lorsqu'une plainte est envoyée au préfet ou au directeur de l'Enseignement primaire, ceux-ci chargent l'inspecteur d'enquêter et de donner un avis sur 
les sanctions à prononcer éventuellement contre l'instituteur incriminé. La tâche des inspecteurs est rendue difficile par la multiplicité de leurs responsabilités. En effet, ils doivent recueillir les témoignages des victimes, des parents, des élèves et instituteurs qui auraient été témoins des punitions corporelles, du directeur de l'école pour établir les antécédents de l'accusé, éventuellement du maire de la commune qui se fait parfois l'écho de l'opinion publique. Ils doivent considérer d'éventuelles circonstances atténuantes, ou aggravantes (notamment la récidive). D’autre part, une sanction exagérée pourrait susciter le mécontentement des instituteurs de leur circonscription. Les inspecteurs s'efforcent en outre de veiller à ce qu'une affaire de punition corporelle ne serve pas de prétexte pour obtenir un déplacement d'instituteur souhaité pour d'autres raisons.

La sanction la plus courante est en effet le déplacement. Dans les quarantequatre affaires étudiées, vingt-six ont pour conséquence la mutation de l'instituteur. Il s'agit d'une véritable sanction, dans la mesure où celui-ci est déplacé sur un poste qui l'éloigne de sa vie sociale habituelle, en général dans un arrondissement périphérique de la capitale ou dans une commune de banlieue plus isolée. Ce changement a pour avantage de faire cesser l'émotion suscitée par l'affaire. De plus, quinze instituteurs ou institutrices sur quarante ont fait l'objet d'une sanction officielle inscrite dans leur dossier professionnel : six reçoivent une réprimande, six un blâme, trois autres sont momentanément suspendus. Deux sont révoqués et doivent quitter l'enseignement.

La position occupée par l'instituteur dans le champ de l'instruction primaire est prise en compte par l'inspecteur quand il propose des sanctions ${ }^{35}$. Par exemple, Jean-Baptiste Bayle, jeune instituteur de banlieue, peu qualifié (il n'est pas normalien et ne possède que le brevet élémentaire), dont les capacités professionnelles sont mises en cause lors des inspections et qui mène, de plus, une vie plutôt dissolue, reçoit une peine de réprimande le 21 juin 1888. Il est déplacé d'Aubervilliers à Pantin, mesure qui retarde son entrée à Paris : en 1890, l'inspecteur note que cette peine disciplinaire ne permet pas de lui donner l'avancement qu'il sollicite, et il n'est nommé à Paris qu'en 1901. Auguste Baudrier, pour des faits de violence plus graves, est quant à lui suspendu un mois et déplacé, en décembre 1880, de la rue du Renard, dans le $4^{\mathrm{e}}$ arrondissement, à la place Jeanne d'Arc, dans le $13^{\mathrm{e}}$.

35 Il est souvent difficile de mesurer les motivations de la sanction et ses conséquences sur la carrière des maîtres sanctionnés. Cependant, lorsque l'ensemble des dossiers de notre corpus aura été exploité, la base de données recensant les informations sur les carrières et l'évaluation pédagogique des instituteurs devrait nous permettre de comparer les parcours des instituteurs ayant fait l'objet de plaintes à l'ensemble de la population étudiée. 
Son écart de conduite s'est produit dans un contexte de tension suscité par la laïcisation des écoles communales parisiennes et le débat sur la gratuité de l'enseignement primaire. Ce contexte, dans lequel toute affaire grave touchant une école parisienne peut avoir des répercussions politiques, explique que Jules Ferry prenne le temps d'approuver, dans une lettre manuscrite adressée au préfet, les sanctions prises contre Baudrier. Cependant, contrairement à Jean-Baptiste Bayle, Baudrier, ancien élève de l'école normale de la Seine où il a été remarqué pour ses idées républicaines et anticléricales en 1875, est considéré comme un excellent instituteur. Ainsi, dès le $1^{\text {er }}$ mai 1881 , les sanctions sont quasiment annulées par sa nomination à l'école de la rue Tournefort, dans le $5^{\mathrm{e}}$ arrondissement.

Quelques mois plus tard, en octobre 1881, Joseph Soyris, ancien instituteur congréganiste, frappe un de ses élèves. La gravité des faits est comparable à celle des actes qui impliquaient Baudrier. Le 26 octobre, l'inspecteur Jacquemart demande donc le déplacement de Soyris, en précisant qu'il a été instituteur congréganiste. Le 30 octobre, il estime qu'il y a urgence à mettre la sanction en œuvre : " les parents de l'enfant qui a été frappé s'agitent, bavardent, pétitionnent pour obtenir le déplacement de ce maître ". Le directeur de l'Enseignement primaire ne se contente pas de cette sanction et demande au préfet la révocation du coupable. Le 14 novembre 1881, Joseph Soyris, niant les faits qui lui sont reprochés, écrit au préfet pour demander sa clémence, mais le ministre approuve sa révocation le 23 décembre 1881. Dans ce cas, le contexte politique joue en défaveur de l'instituteur accusé, en stimulant la représentation du " frère frappeur " - pour reprendre les termes d'un parent d'élève qui accuse en 1888 l'instituteur Joret de " parodier " les instituteurs congréganistes.

Ainsi, l'étude de dossiers des instituteurs et institutrices de la Seine permet d'approcher certaines formes de violence ayant pour cadre les écoles publiques du département de la Seine, tout en les replaçant dans le contexte formé, d'une part, par l'évolution du fonctionnement de l'institution scolaire et, d'autre part, par les représentations de l'usage légitime de la punition corporelle. Ces violences, dans la mesure où elles ont donné lieu à une plainte, laissent entrevoir l'utilisation encore fréquente de punitions corporelles en milieu scolaire, reflet des pratiques encore largement employées par les familles à cette époque, et l'existence d'actes de brutalité plus isolés, qui peuvent parfois s'expliquer en partie par une situation 
de crise de l'établissement scolaire (carence de la direction, sureffectif, tensions liées au contexte politique).

Or, l'institutionnalisation de l'école républicaine est concomitante de l'affirmation d'un refus plus ferme des formes les plus brutales de violence. Les responsables de l'application de la législation républicaine s'efforcent de mettre en œuvre l'interdiction déjà ancienne des punitions corporelles, dans un contexte marqué par la concurrence congréganiste et la volonté de rendre l'institution scolaire conforme aux principes du régime républicain. Aussi, en détaillant les relations sociales qui se sont nouées dans le cadre des établissements scolaires de la Seine aux premiers temps de l'école républicaine, l'analyse historique éclaire une étape de la lente dynamique du changement de l'institution scolaire et de la société française vers le rejet du recours à la violence dans tout processus éducatif.

Jérôme KROP

Doctorant, université Paris-Sorbonne-Paris IV krop.jerome@wanadoo.fr 